\title{
MediFrame: A Tablet Application to Plan, Inform, Remind and Sustain Older Adults Medication Intake
}

\author{
Lea Gulstav Dalgaard \\ Pervasive Healthcare \\ The Alexandra Institute \\ Copenhagen, Denmark \\ lea.gulstav@alexandra.dk
}

\author{
Erik Grönvall, Nervo Verdezoto \\ Department of Computer Science \\ Aarhus University \\ Aarhus, Denmark \\ \{gronvall,nervo\}@cs.au.dk
}

\begin{abstract}
Healthcare activities in private home settings that are not supervised by a healthcare professional can challenge older adults. To support such unsupervised care activities, an increasingly number of reminders and monitoring systems are designed. However, most of these systems target a specific treatment or a specific condition. In this paper, we discuss experiences from designing the tablet-based application MediFrame. MediFrame is a personal medication management system to support older adults in non-clinical settings such as the home. The paper describes the user-centered design process and the resulting tablet application. We show how MediFrame can be used to support adherence in medical treatments through fieldwork informed use scenarios. Based on early qualitative feedback, we also discuss lessons learned and how designers can support a holistic medication experience for an older adult's everyday life.
\end{abstract}

Keywords- medication management; design; tablet; older adults; user-centered design

\section{INTRODUCTION}

The World Health Organization (WHO) [1] reports that adherence to long-term therapies is around $50 \%$ in developed countries and even lower in developing countries. For instance, about half the people subject to prescribed medication fail to take their medications accordingly $[2,3]$. In the US, medication related problems had a cost of approximately $\$ 177$ billion in 2001 alone [4]. According to the same study, this cost was doubled from 1995 to 2001. Better adherence to a prescribed treatment together with documentation of use and side effects is one way to help both the individual and society to control this cost.

Institutional care is more resource-demanding compared to home-based care [5] and moving from an independent to a dependent life situation is a major concern among people over the age of 60 [6]. To address this, the healthcare system tries to enable citizens to remain self-sufficient. A prolonged independence can reduce healthcare costs while helping people to sustain a dignifying, independent lifestyle [7].

The Danish welfare state provides free healthcare while motivating citizens to take responsibility for their own health and lives [8]. A new, Danish, national funded initiative is the Shared Medication Record (SMR) [9]. It provides physicians and care professionals at different locations such as hospitals, the general practitioners out-clinic and municipality care workers with access to all people's prescribed medication. SMR is also accessible to people through the Internet. In the project, we examined how to support older adults in their everyday medication management. We developed user-centered extensions, or add-ons, to the SMR. Our work leverages previous research that demonstrates how electronic medication monitoring data can benefit pharmacists and physicians as it empowers them to adjust patient treatment plans [10].

This paper presents the iPad-based prototype MediFrame that aims to postpone the transition across care settings by supporting people's everyday medication intake. In the following, we present related work, our user-centered design process with older adults and medication experts, the MediFrame application and its early evaluation phase. The main contribution is the presentation and discussion of the design of mobile medication management support for older adults in everyday life. Finally, we present a MediFrame use scenario and discuss lessons learned regarding medication management support for older adults in everyday life.

\section{RELATED WORK}

\section{A. Mobile Technology and Older Adults}

The use of smartphones and their built-in sensors has become widespread when supporting older adults' daily activities. However, one disadvantage of mobile phones may be the limited screen size when an older adult is expected to use screen-based interaction [11]. Here tablets with touchtechnology can be an alternative $[12,13]$. Research has also shown that touch screen interaction is more suitable for computer-novice older adults rather than using keyboard or mouse [14]. Furthermore, touch-based interfaces suite activities that should be integrated into people's everyday lives [15]. Touch interfaces can be easy to learn and adopt by older adults besides their physical or cognitive capabilities [15]. In addition, tablets can also fit into the sometimes limited space of older adults' homes [12].

Indeed, integrating healthcare technology into older adult's homes can be challenging [16]. People tend not to put too much emphasis on their illnesses while at home, and assistive technologies that prevent stigmatization are preferred by many users [17]. When designing healthcare technology for the home, it is important to consider how people arrange things and activities at specific places to maintain the order of the home [18]. In this sense the aesthetics of the home plays an important role [18] and tablet 
cases, covers and sleeves can provide several opportunities to allow a more personal and aesthetical fit in private homes. Modern, mobile technologies, such as tablet-computers can prevent stigmatization through its design and additional 'non-healthcare use'.

\section{B. Technology for Medication Management}

A set of physical and digital artifacts are available to support people's medication intake, such as reminders, pill boxes, software applications, tablet-holders, automatic medication dispensers and paper-based medication lists [19]. An example is the SmartPill web application [20]. It presents the medication level in the blood and can send SMSwarnings if a medication dosage have not been taken in time. MoviPill [21] creates a persuasive, medication adherence game for older adults by combining mobile technology and pillboxes. Others examples of augmented physical objects for medication management are MedTracker [22] and the smart pill box [23]. SmartDrawer combines a physical drawer with a RFID-based reminder functionality [24]. GlowCaps [25] is a medication container cap that uses light, sound, or telephone calls to remind a user to take medication and calls if a refill is needed before the prescription runs out. GlowCaps also provides a monthly adherence report to family members or the general practitioner.

Apps for the iPhone can for example provide medication list management and overview [26] or setup and receive medication intake reminders [27]. Most of these systems use a generic medication image (i.e. an iconic pill), but [28] allow users to download the actual image of pills or take photos of their own medication.

\section{Patient-Physician Collaboration and Information Sharing}

How to enable an effective patient-physician communication has been widely studied [29-31] and recognized as a key factor in increasing treatment compliance $[1,30]$. To successfully carry out agreements, the collaborative partners rely on a shared intention within the group [32]. The facilitating technology can be seen as one actor in such collaborations. At clinics, information exchange is composed by information seeking, documentation and patient education [33]. Using modern telecare solutions, communication can include locations outside of the clinical setting [34] and mobile phone-based diaries have been used to support for example everyday diabetes management [35].

\section{Design Implications for Medication Management}

Design implications for medication management systems include (from [36]): 1) to pay special attention to placement, readability, and terminology of instructions and confirmations; 2) to prioritize performance over preference when designing interfaces for personal health applications; 3) to present individual's health information by finding a balance between textual information and design metaphors; 4) to provide automated mechanisms to perform basic medication management tasks but requiring minimal interaction steps; 5) to consider all stakeholders to address possible conflicts and avoid misunderstandings between each stakeholders; 6) to explore and understand individual's unique practices for health information management; 7) to provide the possibility to add more functionalities as users master the basic ones; (and from [37]) 8) to provide customizable reminders; 9) to facilitate information search; 10) to recognize the value of portability for the reminding; 11) to allow users to retrieve information about the context; (and from [23]) 12) to provide low-level details regarding an individual's behavior; 13) to allow collaborative sharing and exploration among members of the care network; 14) to support annotation of health-related information before sharing to avoid misinterpretations; (and from [38]) 15) support distribution and personalization according to spatial arrangements that sustain routines; 16) consider privacy and ethical considerations; 17) consider a broader scope of health to extend the context of a patient-physician interaction; and 18) provide a mechanism of inference for remote assistance.

Our work emphasizes the need to support the medication information exchange across care settings. We focus on both the documentation of medication intake and the understanding of people's medication information seeking needs at home. To do this, we build upon Bates' model for information seeking behavior [39] composed by: a) active information seeking including browsing (undirected active) and searching (directed active); and b) passive information seeking including monitoring (directed passive) and being aware (undirected passive). By facilitating people's medication information seeking, MediFrame aims to support the medication information exchange, especially when people are away from the clinic and their physician.

\section{DESIGNING FOR MEDiCATION MANAGEMENT}

First, the project initially carried out a quantitative study with 316 older adults over 60 years of age and a qualitative study with 9 older adults (aged 60-93) [19]. This initial fieldwork revealed that the lack of knowledge about medication, forgetting the medication intake, complexity of the medication regimen, taking medication outside the home, the lack of support for caregivers, and substitution of medication are important challenges for older adults.

Second, we reviewed existing mechanisms and technologies used to support peoples' medication intake. Our review included electronic reminders, tablet holders and dosing aids with electronic reminders, automatic dispensers, and non-electronic tools such as paper-based medication lists and pillboxes [19]. We found some of these artifacts in older adult's homes. These technologies might support people in different ways but also present disadvantages such as lack of usability and knowledge regarding people's daily activities (routines or not), and a too generic support (e.g. reminders, pillboxes) that might not be enough for people with a complex medication regimen. Based on our initial fieldwork and this review, we defined a set of functional (integration, reminder, tracking, mobility, medication information search, sharing, flexibility for systematic storing of medication, control) and non-functional (security, safety, performance, privacy, user interface) meta-requirements for the design medication management systems [19]. 
Third, MediFrame was designed following a usercentered design process [40]. This process involved older adults, medication experts and researchers. Eight older adults (aged 57-90) and seven medication experts participated in several workshops (two-three hours' sessions). The medication experts were two doctors, two healthcare workers, two pharmacists and a developer from SMR. The MediFrame design team was informed by the initial user meta-requirements and the related work in which tablet technology seems to provide several opportunities for homebased healthcare technology. Workshops together with the older adults involved 1) discussions about how participants manage their own medication, the amount of medications and the frequency of the medication intake, strategies to remember a medication dose, current technology to support the medication intake and their need of medication information, 2) identification of older adult's daily routines including their medication intake by filling a paper-based weekly calendar, and 3) early feedback regarding the functionality and goals of MediFrame based on early prototypes. Workshops together with medication experts involved 1) discussions about the results of the workshop activities with older adults, 2) gathering knowledge regarding medication information for example the exact definition of a dosage, the use of generic and active substances and the right phrasing for a medication dose, and 3 ) feedback and suggestions on the functionality, vision and goals based on early and revised prototypes of MediFrame. Additional meetings were hold between the researchers and the medication experts to further explore and confirm specific situations that emerged from previous activities

All design decisions were based on the results and feedback from the workshop activities. Now, we further describe our initial list of requirements and design process.

\section{A. Initial MediFrame List of Requirements}

Based on the related work and the tablet technology the project defined the initial list of requirements for MediFrame. We focused on a specific set of the aforementioned meta-requirements [19]:

1) Integration: MediFrame should integrate validated medication information from trustable sources (e.g. the "patient information leaflet"). This also implies an automatically update of prescribed medication (by doctors) and handed out medication (by pharmacist) to provide information about substitute medications.

2) Reminder: MediFrame should provide customizable reminders to each person's medication regimen and in accordance with their daily activities including the medication refill reminder.

3) Tracking: MediFrame should document the patient's medication intake or lack of it over time. This tracking information includes issues about intentional non-adherence and regarding the current medication regimen such as side effects or reasons for skipping a dose.

4) Mobility: MediFrame should support medication intake in and outside the home especially when daily routines are broken.
5) Search: MediFrame should facilitate an active medication information search from trustable sources (e.g. "patient information leaflet").

6) Sharing: MediFrame should sustain caregivers to play an active role in the user's medication intake.

7) Control: MediFrame should let the users decide when to start or stop sharing their medication information and receiving reminders.

8) Privacy: MediFrame should allow users to preserve their personal dignity by giving them control over functionalities that can rise privacy concerns such as reminders and sharing information.

9) User Interface: MediFrame should be able to support several doses of medication per day with different type of formulation considering the capabilities and limitations of tablet technology.

\section{B. Exploring the design space}

We initially investigated several metaphors such as the "medication cabinet" and the "clock" to explore possibilities of arranging medications and reminders [19]. We used a number of early low fidelity prototypes [41] to elicit discussions and get early feedback from participants. These prototypes were manifested in several design drawings and mock-ups that in combination with the tablet device allow us to get a rapid user feedback on our MediFrame concept. We additionally reviewed existing guidelines for mobile devices regarding touch interfaces and older adults to frame our design space. A total of 31 design guidelines were classified as guidelines for visual and interaction design (17), information organization (4) and conceptual consideration (10) that can be adapted for tablet-touch interfaces [42].

\section{Iterative Prototyping}

During our design iterations, the low fidelity prototypes moved from early sketches, to screen-based prototypes and finally into a more interactive high fidelity prototype. Figure 1 shows an example of a low-fidelity prototype during test. To support user's information seeking, we based some designs on Shneiderman's visual information seeking mantra "overview first and details on demand" [43] to avoid the visual clutter for older adults.

Applying Bates' model, the workshops focused on understanding how people relate to interface features and information (e.g. phrasing, icons, layout, navigation).

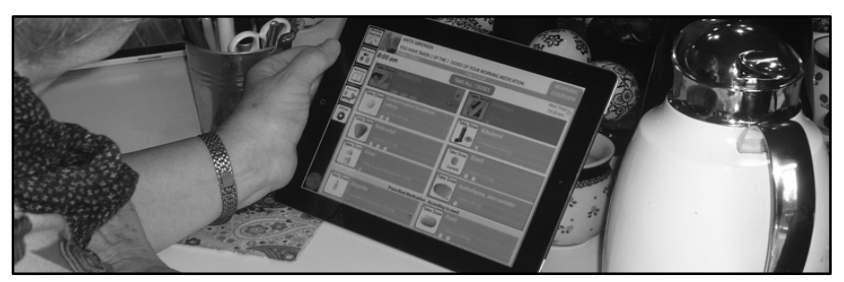

Figure 1. Testing our screen-based prototype

Bates' model was applied to MediFrame during the iterative design process to discuss and revise previous interfaces relying on user's information seeking behavior. 


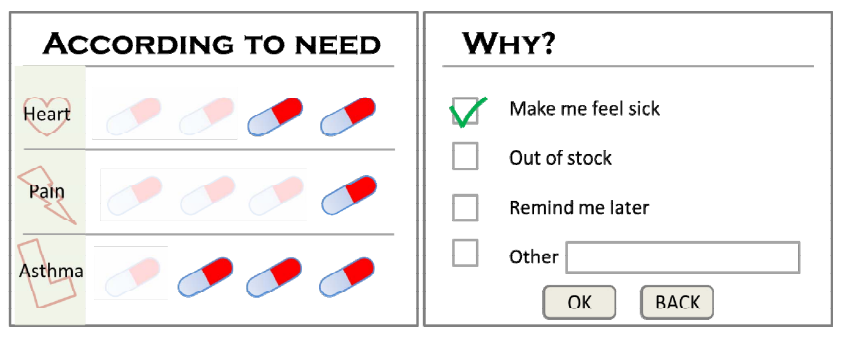

Figure 2. According to need (left) and registration of non intake reasons (right) mockups

\section{Additional Requirements}

A number of unforeseen challenges emerged through our design work, for example the case of the so-called "according to need"-medication [40]. According to need medication is medication that you do not take according to a schedule, but rather when there is a perceived need. Painkillers can for example be prescribed as "according to need"medication. During our user workshops it was revealed that the older adults did not perceive this type of medication as 'real' medication and therefore they did not keep track of it. Also, the older adults did not document why they did not take a medication. The medication experts emphasized the importance of getting this sort of information from the older adults, to better adjust the prescribed regimen. These specific challenges were further discussed with both the medication experts and the older adults and investigated using low fidelity prototypes as illustrated by Figure 2 .

After a 10-month user centered design process, our empirical study suggests five design principles that account for medication specific particularities (according to need medication, the heterogeneous care network, the substitute medication, the medication informational order, the shared responsibility and the adjustment of medication intake) in practice for everyday medication management [40]. Then, we initiated the high fidelity prototype development phase.

\section{The MediFrame ApPlication}

Informed by the related work and medication specific particularities [40], MediFrame aims to support unsupervised everyday medication intake allowing users to 1) plan their medication intake on a day-to-day basis. Also considering other scheduled activities such as work or leisure; 2) retrieve information about each medication they are prescribed; 3 ) get reminders when to take their medications; 4) get documentation and support in their medication-related dialogue with their general practitioner, and 5) support the intake of "according to need"-medication.

In addition, MediFrame relies on the user's active search of medication information. When applying Bates' model to MediFrame; prescribed, substitute and "according to need"medication are considered main, directed information. And relevant medication information such as side effects or instructions is considered undirected information.

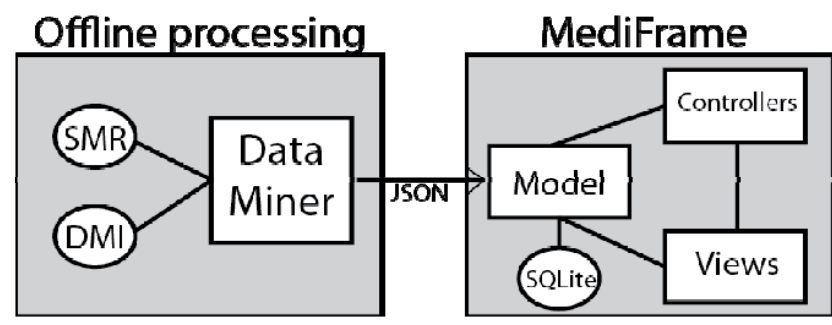

Figure 3. MediFrame System Aarchitecture

As stated before, the project has the national, upcoming Shared Medical Record (SMR) service in mind. Even if the medication information is available to the citizen through a web service, the SMR's security model does not at this point allow a seamless mobile integration. Hence our application works with offline data records, identical to the online version of SMR. In addition, MediFrame integrates a Danish Medication Information (DMI) service, made available online by one of the project partners [44]. This service allows us to show images of each commercially available medication in Denmark and collect information such as the medication's effect, possible side effects and when and how it should be taken (i.e. the Patient Information Leaflet).

\section{A. MediFrame System Architecture}

MediFrame is a web (cross-platform) application based on the Model View Controller (MVC) design pattern. It uses the Sencha Touch Framework [45] to access native device APIs and is embedded as a native shell (using PhoneGap [46]). The cross-platform approach allows MediFrame to be deployed on other mobile devices and platforms in the future, but is currently being evaluated using the Apple iPad.

Two main components describe the system architecture of MediFrame: 1) the offline processing, and 2) the MediFrame App (see Figure 3).

1) Offline processing: It is necessary to preprocess and re-structure the data that comes from the two Danish services: SMR and DMI. This is managed by the Data Miner. The Data Miner generate JSON files to instantiate the data model for the MediFrame App.

2) MediFrame App: The MediFrame App is the tablet application developed using the MVC design pattern. The status and the application's data are stored using SQLite in the tablet device. Furthermore, the application defines and combines the controllers, views and models to provide the user with different functionalities. Each functionality has its own controller and view classes (e.g. CalendarControllerCalendarView) except from the main Controller Application which manages the MainView of the application, the TakeMedicineView and MoreInfoView.

\section{B. MediFrame Functionalities}

As a result from the cooperative design process, MediFrame implements the following functionalities:

1) Calendar: This function provides an overview of the scheduled medication intake (see Figure 4) in relation to other planned activities (e.g. a private or work agenda). People can adapt and personalize their medication intake based on this information. For example, the user can 'at a 
glance' identify medication and activity overlaps that can challenge adherence. The user is also allowed, within given timeframes, to adjust and reschedule the medication intake to suit his/her everyday activities, especially when routines are broken. The interface provides different levels of aggregation: per day, week and month. It also provides details on demand by selecting each medication from the calendar. In addition, it can import events from personal calendars (e.g. google calendar). We implemented the calendar using the FullCalendar Javascript library [47].

2) Medication: This function makes the user and potential caregivers able to get an overview, and retrieve information, about all prescribed medication. The medication information (i.e. the Patient medication leaflet) and images are retrieved through an online service (DMI) to be used by the DataMiner (see Figure 3). The medication list can be filtered, providing different views for example "today's medication" or "all prescribed medication". It provides details on demand by selecting each medication.

3) Diary: This function allows the user to document information about the medication and experienced side effects in a diary-style log. It also provides a mechanism to automatically log several medication-related events such as an updated medication list, reminders, and medications not taken according to plan. Hence, it provides an overview of these events during the medication administration activity.

4) Reminder: This function provides users with medication reminders when it is time to take medication or a refill is needed. A reminder can be set to be active (sound and visual) or passive. In passive mode, MediFrame presents itself as a digital picture frame and the remindersignal is a specific picture that appears on the screen (e.g. a picture of a flower). Seeing the signal (i.e. the flower) the user taps the screen to start the interaction with MediFrame. The specific cue-image is repeatedly displayed on the screen until the user takes action.

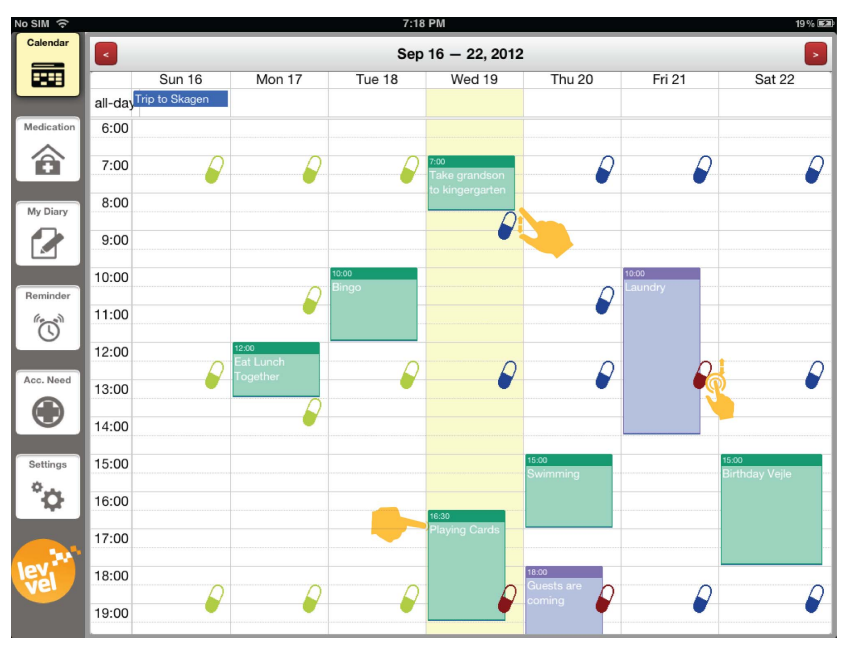

Figure 4. The Calendar Interface. The Wednesday morning medication have been moved to suit planned activities and the evening medication overlaps with a planned activity

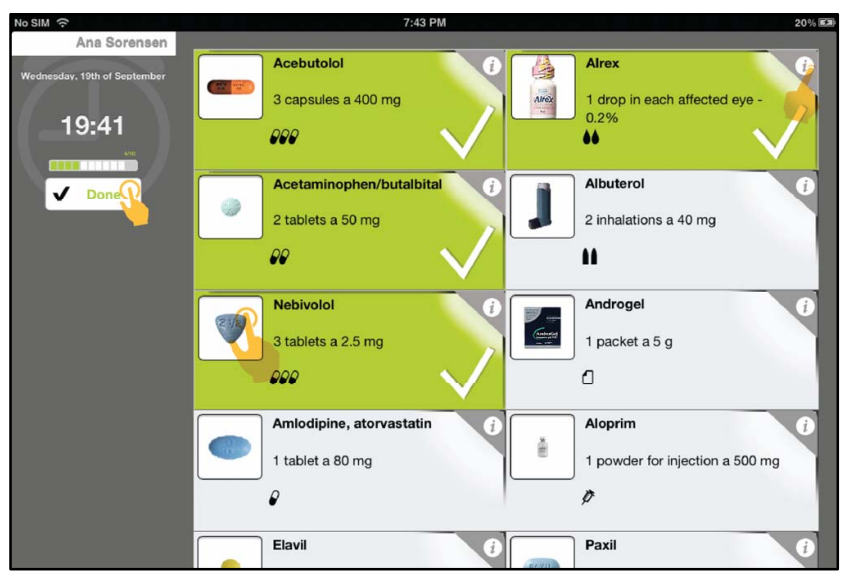

Figure 5. The "Take Medicine" Interface. Currently four out of ten medications have been taken

5) According to Need: This function allows the user to document the use of 'according to need'- medication. Here the user starts by selecting a symptom or condition rather than a medication (see Figure 2). MediFrame then informs what prescribed medication to use in this particular situation. The registration of 'according to need'-medication is also documented in the Diary.

6) Settings: MediFrame allows the configuration for some of the above-mentioned functions. For example, it allows the user to personalize each medication's reminder respecting the limitations imposed by each medication. For instance, organizing all morning medications by selecting the prescribed medication according to the specific time frame. Helping users to set reminders and medication time according to their own needs.

7) Take Medicine: This function allows the user to register the medication intake at specific times a day by tapping each medication button (see Figure 5). When a user taps the ("I")nformation icon, relevant medication information is displayed regarding the selected medication. It also allows the user to manage potential substitute medication by highlighting this medication in the interface. All selected medication are clearly displayed on the interface. Furthermore, a confirmation step is added by a "Done" button replacing a prior "Take all doses"-button from earlier versions. In addition, the "progress bar" provides feedback about the status of the current medication intake at hand. When tapping the "Done"-button, it provides a motivational feedback if all scheduled medications have been taken accordingly, otherwise, it asks the user to register (by selection) the possible reasons for skipping the current dosage as presented in Figure 2.

\section{EARly EVAluations}

\section{A. Concept Validation}

An initial MediFrame concept validation was performed together with the medication experts and four older adults [19]. They stressed that simplicity is the key to embrace as many diverse users as possible and that users might not want or need too much information on the screen. While concerns 
emerged that MediFrame could be difficult to use by people with motor impairments and dementia, the evaluation team stressed that in such cases MediFrame could be useful for the healthcare professionals and care providing family members.

Based on the feedback, we found that older adults rely on the use of images (e.g. visualizing two graphical medication icons instead of writing 'take 2 pills') and medication experts suggest providing user's identification (i.e. the user should have his/her name clearly written within the interface - this is especially important when more users, e.g. a couple, share the tablet) as main features of the interface.

\section{B. Testing the Screen-based Prototype}

The medication experts were concerned about how to visualize and support a complex medication intake, for example when a large number of different medications should be taken at the same time. We decided to address this complexity on the "Take Medicine" interface by displaying up to 10 prescribed medications at the time, providing a balance between information granularity and overview. After an iterative user-driven refinement of our mockups, we performed a user test of the "Take Medicine" interface with older adults using a screen-based prototype as illustrated by Figure 1. We visited three older adults and asked them to explore our interface using the iPad tablet. We provided an introduction to the tablet technology prior to the user test.

Based on the participant's feedback, new issues emerged. First, users got confused having more visible functionality than needed. Second, participants tend to press the "Take all doses" button without pressing all the medications in the interface. Third, the screen-based prototype embedded interaction-limitations that confused our users. Fourth, the redundant information provided by a combination of textual information and visual representation (i.e. a picture of three pills rather than showing the number 3 to indicate the current dose) was preferred over the textual representation. Finally, the interface features such as font size, text and buttons were well accepted by older adults as they could distinguish for example all selected items or recognized buttons by their appearance (button-perceived affordance).

\section{An Evaluation of the High Fidelity Prototype}

Considering the results of the previous evaluations, we now started evaluating the current high fidelity prototype focusing on the understanding of user's information seeking needs. We visited an older adult to get an initial feedback from our "Calendar" and "Take Medicine" interfaces. She was 87 years old and she has never used a tablet device before. First, we asked the participant to write down her weekly activities and her prescribed medication on a paperbased calendar. We used this information to configure the Calendar to represent a real situation. During the test, we evaluated both the Calendar and "Take Medicine" interfaces.

1) Calendar Interface: The calendar interface was perceived as a good overview of scheduled activities and prescribed medication (directed information). However, we observed that the participant did not detect the scrolling possibility within the interface while seamlessly working with the other interface elements, such as buttons, etc. Furthermore, the undirected information in the calendar such as the status of the prescribed medication (i.e. whether it is planed, taken or 'in conflict' with another activity) is perceived as useful information. Regarding a possible conflict between a planned activity outside the home (e.g. playing cards) and the scheduled medication, the participant said "It would be very useful to know from this[MediFrame] that I should take it [the medication] with me".

2) "Take Medicine" Interface: The "Take Medicine" interface was perceived as a good overview of prescribed medication that should be taken at a specific time of a day (directed information). The participant understood the name and dosage of each medication. However, she got confused when she noticed one incorrectly written medication in the interface. This shows the complexity with for example handling subsititute medications that regularly can change names. Furthermore, the undirected information in the interface, such as side effects and what the medication is for, which appear when the user presses the additional medication ("I")nformation icon, was perceived as important. The participant said: "If I have doubts about it... if I shouldn't take to many of them then I would look at it [("I")nformation icon] to find out if it is not good to take it more than once a day". The participant stressed that information regarding how long a person should take a medication, and whether it is temporaly or not is desirable. She said "it would be nice to know... if you have to change it [medication] after a while or if I should continue for the rest of my life".

As a result, it was clear that MediFrame relies on the user's active search and browsing of information. In general the results were positive regarding the overview of information. The participant said "I think this [MediFrame] is very good even thought I am against technology...”.

\section{MediFrame Use ScEnARio}

We developed envisioning and use scenarios during our design process to explore and validate our concept. The MediFrame functionality, to a large extent, enables these scenarios. We present a use scenario based on experiences from our design process to illustrate the use and benefits of MediFrame.

It is early Tuesday morning and Ann wakes up and has to take her Alendronat (bisphosphonate) for her osteoporoses half an hour before she eats her breakfast. Ann wakes up around 7 o'clock every morning and has configured MediFrame to remind her to take her medication immediately when she wakes up. MediFrame opens up the 'Take Medicine'-view. Ann confirms taking the Alendronat by tapping on its icon. If Ann makes a mistake, for example tapping the wrong medication she can simply 'undo' the action by tapping the erroneous selection once again. Now Ann concludes the activity by tapping "Done" as no other medication is scheduled now. MediFrame plays a comfortable melody to confirm the action, which makes Ann confident in her medication intake.

Later today Ann is going to the elderly centre to play bingo from 10 to 12 AM. Today Ann and her friends are 
staying at the centre afterwards to eat lunch together. Before she leaves her home she opens up the MediFrame Calendarview and becomes aware that she should bring her noonmedication (for her back pain and diabetes) as her daily activity and scheduled medication are overlapping. Ann collects her noon-medication in her purse and leaves for the elderly center.

In the evening Ann's son Thomas and her grandchildren are visiting for dinner. Thomas is cooking dinner. Ann notices a head of cabbage and recalls some complications eating cabbage with her daily intake of anticoagulant medication (Marevan). She activates the Medication-view where all her medication is listed. Here Ann can see a detailed description of each medication, when to take it, how much to take, side effects and what kind of food/drinks she should avoid when taking it. Ann selects Marevan and a part of its patient information leaflet is presented and confirms that she should not eat cabbage because it contains vitamin $K$, which reduces the effects of the anticoagulant medication.

The grandchildren are having a sleepover at Ann's house the same night, which means that Ann spends some time finding the mattresses and making their beds. Because of this breakdown of her normal routines, she questions whether she took her evening medication after dinner or not. MediFrame can help Ann to recall her medication intake as this is registered in the Diary-view. Hence, she checks My Diary that confirms she indeed has taken her evening medication. MediFrame makes Ann feel secure in her daily medication management and she can go to sleep not worrying about it.

The presented scenario illustrates several challenges in Ann's medication experience. In addition, the scenario shows how MediFrame can support Ann's medication intake especially when the routines are broken. We now discuss and present the lessons learned during our design process.

\section{DISCUSSION AND LESSONS LEARNED}

Based on our observations and the input gathered during our design process, we will now discuss lessons learned during the MediFrame design process.

\section{A. The Use of Tablets by Older Adults}

MediFrame facilitates the transition between the clinic and the home by supporting people to manage their daily medication intake. The older participants in our study did not previously use smart phones and tablets. However, all were positive about using a tablet that can help them gain more information about their medication. So far, they have used pillboxes, reminders and paper-based forms to track their prescriptions. Medication experts expressed a positive feedback as MediFrame can be seen as a tool that can mediate the communication between care settings. They emphasized that it is useful to know what is happening with older adults at home between consultations. One nurse commented that "it [MediFrame] can be a way to know why they are not taking their medication as we [nurses and general practitioners] don't know why they come back after few weeks of discharge from the hospital'.
1) Introduction of Tablet Technology: It turns out to be a good strategy to give older adults an introduction to the iPad technology - how it works and how they can use it - before the test. This introduction helps us to discover their attitudes towards the technology as such. In one of our workshops testing a screen-based prototype, one participant (woman, 90 years) kept her distance to the tablet at the beginning of the activity. But after receiving the introduction she was curious about the tablet and engaged in our workshop.

2) Guidelines for Touch Interfaces: We have considered existing guidelines for designing touch interfaces provided by: 1) related work areas such as user interface design, mobile interface design and usability and accebility for mobile devices, 2) specific work on touch-based interfaces for older adults, and 3 ) guidelines to design for older adults [42]. However, no previous work has suggested how to improve the design of tablet-touch interfaces that target older adults. Consequently, we have adapted the existing guidelines and validated some of them (e.g. "do not repeat the navigation...") to frame our design space. For instance, we decided to remove the main menu from the "Take Medicine" interface after the screen-based evaluation as users got confused having more functionalities than needed. Unsurprinsingly, conceptual considerations such as "keep the user in control", "good dialog design..." and "reduce short-term memory and cognitive loads..." [42] also apply for the design of tablet-based interfaces for older adults, but their application get a more important role in the healthcare context. For example, we changed the "Take all doses" (i.e. medication) button to a "Done" (i.e. task complete) button to add a confirmation step and avoid the laziness of pressing the "Take all" button without selecting all medications one by one. This concern was also discussed by our medication experts who support our design decisions.

We suggest that a further formal validation of the existing guidelines should be iteratively done through the use of prototypes to suit and design for older adults. In addition, a short, but concise, introduction to tablet technology and its use should be provided to users that have no previous experience of such technology (as our older adults) to enhance their participation in user tests.

\section{B. The Taking Medicine Interface - Simplicity is the Key}

As mentioned in section 4, the MediFrame design involved different actors during the design process. In particular, medication experts were concerned about the complexity of the medication regimen. They emphasized that a clear overview that contains all medications for a specific time should be visible to older adults on the screen. Previous work has applied the medication clock metaphor for medication management on a mobile device such as CollaboRhythm [48] and Colorado Care Tablet [36]. However, we found that the clock metaphor might be challenged due to the complexity of our older adults medication intake [19]. Indeed, the older adults in our study are taking up to 35 medications distributed across the day at different times. This complexity challenged our interface design as we moved away from our initial idea of metaphors as also suggested by [36] through the use of the diverse low- 
fidelity prototypes. In addition, the medication experts stressed that simplicity is the key and that users might not want, or need, too much information on the screen.

The "Take Medicine" interface provides directed, active, medication information (search) by giving an overview of a specific time's medication dosages at a glance. This facilitates the registration of the medication intake. An undirected active medication information (browsing) is provided by giving access to more relevant specific medication information by selecting the ("I")nformation icon. This can support the medication intake by providing relevant information such as instructions of how to take a medication. Directed passive medication information (monitoring) is provided by the overview where the selected/taken medication changes color and a progress bar provides overview. An undirected passive medication information (awareness) is provided by medication reminders and warnings as a user wants to skip a medication.

1) The use of images: After our first users' feedback (older adults and medication experts), we found that older adults rely on images regarding their medications. A medication expert said, "you [older adult] don't need big pictures of the drug in this overview. Small size may be good enough". Even though the use of images helps users to identify medications, the complexity of the medication intake was not addressed in early prototypes.

2) Addressing the complexity of the medication regimen: Medication experts see MediFrame as a medication checklist that can help them to track and remember whether or not the older adults have taken all medications for a specific time, specially during a complex regimen. Based on the Shneiderman's visual information seeking mantra [43], we provide a medication overview where older adults need to register their medication intake. Considering the medication complexity and medication experts' feedback, we defined a list with up to 10 medications that can be displayed for a specific time on the screen. For instance, one of our older adults took 5-6 different pills, 5-6 times a day. MediFrame is expected to support her medication intake.

3) Providing user's identity: Medication experts expressed that "it is very important to see the name of the person which the information concerns". Especially if the system can help more than one person. For example, spouses play an active role and there are cases in which the couple are taking the same medications (i.e. diabetes medication) as supported by our empirical material.

4) Facilitating the information seeking in the interface: Considering the feedback from the older adults and the discussions together with the medication experts, we took several design decisions to improve the visual information seeking. For example, the main menu was removed from the "Take Medicine" interface, a combination of textual information with a visual representation of the number of medications to take for a specific dose was provided, and a clearly distinction of all selected medications was applied.

5) The use of Low- and high-fidelity prototypes: The early paper-based prototypes supplied user feedback and helped us to identify different design problems. The early prototypes also challenged our participants as they had problems separating the MediFrame vision from the embedded limitation in the early prototypes. For example, they found that a tablet reacted "incorrectly" to their actions, as the system was not fully developed.

We suggest that a medication overview that supports the registration of user's medication intake should be designed to support the four modes of information seeking behavior at a glance (i.e. no scrolling required): browsing, monitoring, searching and awareness. Designers can follow the information seeking mantra by applying and validating existing and generic guidelines for user interface to provide a medication intake overview at a glance. By doing this, designers can work around the technology constraints such as the limited screen size of a tablet device to find a balance between complexity and information seeking needs.

\section{The Calendar Interface - Planning and Reminding the Medicaton Intake at Home}

MediFrame aims to help people plan their medication intake by combining information across care settings. First, MediFrame uses data from the Shared Medication Record [9]. This information is currently generated at a clinical setting. Second, it uses data from people's scheduled everyday activities that is generated in non-clinical settings.

Based on our fieldwork, we have encountered participants that do not have well-established everyday routines. Recalling Ann's use scenario in the previous section, there are active older adults that have activities outside the home for example at the activity center. One participant showed us her agenda in which she writes all her appointments such as visiting friends, consultations, trips, and church visits. To provide medication awareness, monitoring, browsing and searching according to Bates' model [39], the Calendar interface provides an overview of the current and daily medication intake combined with people's scheduled activities (see Figure 2). The calendar provides directed active medication information (search) by giving an overview of several dosages of medications during the day or a week. This facilitates the planning and adjustment of medication intake when Ann needs to go out. An undirected active medication information (browsing) is provided by giving access to the specific medication information (e.g. what is this medication used for?). This information is provided when users search for that knowledge and tap of specific medication. Directed passive medication information (monitoring) is provided by the calendar using different colors for the medication icon. This helps users to remember whether or not they have taken a medication, and follow up their medication intake or look for conflicts between medication intake and planned activities like the red pill icon on the screen. Finally, undirected passive medication information (awareness) is provided by the calendar by giving warnings for possible conflicts between future medication intake and scheduled activities apart from the red color pill. The participant also experienced small problems regarding the scrolling in the calendar.

We suggest that a calendar interface for medication management should be designed to provide an overview of medication intake at a glance (limiting the scrolling as much 
as possible) that supports the four modes of information seeking behavior: browsing, monitoring, searching and awareness. In doing so, MediFrame (or another similar application) can improve the visual perception and the information sense-making process. The overview can later be used as a cognitive artifact [49] that allows people to get a complete overview to recall a medication intake, plan (e.g. move and adjust a medication dose) and manage (set reminders) possible conflicts or breakdowns between medication intake and planned activities. Indeed, automated reminders of daily events and calendar mobility within and outside the home have been reported as important features to support calendaring routines at home [50].

\section{Documenting and Providing an Overview and Knowledge about Medication at Home}

As illustrated in Figure 5, the "Take Medicine" interface registers 1) the medication intake, and 2) information why a user might have skipped a dose. This can inform a later dialogue between the individual and the physician. During our evaluations, medication experts expressed the necessity of presenting the right additional information or instructions when supporting the current medication intake. While the Patient information leaflet is always distributed together with the medication, this information is not always available at hand. The leaflet might have been thrown away, misplaced, or not always easily to understand by the reader. The leaflet text is often rather small, something that can also challenge an older adult in comprehending the text.

We suggest that a medication overview that supports the registration of medication intake should be designed to integrate, and visualize, relevant parts of the medication leaflet in an understandable manner. To do this, it is important to get an understanding of the user's information seeking needs in order to apply the information seeking mantra on the interface. By considering user's information seeking behavior, designers can provide a more complete and fresh experience-based interaction schema as suggested by [51]. Moreover, helping users to document all the specific medication particularities at home and in practice can support physician's information seeking according to specific care plan. This documentation can bridge the gap between laypeople and health professionals that exist between consultations due to the lack of knowledge about what is happening at home during a medication treatment.

\section{CONCLUSIONS}

We have presented the table-based reference implementation MediFrame. Naturally, an implementation of medication support can take many other forms and MediFrame represents only one attempt to interpret and work with a challenging group as older adults. As MediFrame is not a disease-specific application, MediFrame targets most medication situations including 'according to need' and substitute medications.

Our cooperative design process with older adults and medication experts helps us to define the main functionalities (calendar, medication, diary, reminder, according to need, taking medicine) for such a system, aiming to support medication management activities at home. We have described how MediFrame implements two of these functionalities in detail (calendar and taking medicine) and our lessons learned during the 10 months design process. The existing design guidelines that can be adapted for tablettouch interfaces need to be further validated with users to see how the visual information seeking can be improved but getting a better understanding of the user's information seeking needs. These strategies can help designers to improve tablet-touch interfaces for older adults supporting a holistic medication management activity and facilitating the information exchange between patient and doctors.

We argue that it is beneficial that a system automatically updates the individual medication; however, this automation provides new design challenges. For example, substitution medication handed out by the pharmacy can make the automated update process more complicated as the provided medication is different from the one written on the original prescription. While much research is still needed, this paper has shown the importance of looking beyond single strategies and solutions targeting a specific subset of users or diseases. Rather, there is a need to develop a more complete understanding of user's medication information seeking needs in everyday life.

From our perspective, future work includes a more extensive evaluation of MediFrame and to investigate novel ways of working and implementing, integrated solutions to support older adults' everyday medication management.

\section{ACKNOWLEDGMENT}

We would like to thank our colleagues and all participants, especially the elderly citizens involved in our project and the Lev Vel Consortium for their cooperation. Lev Vel is funded by The Danish Council for Technology and Innovation and The Capital Region of Denmark.

\section{REFERENCES}

[1] E. Sabaté, Adherence to long-term therapies: evidence for action 2003: World Health Organization.

[2] B.R. Haynes, E. Ackloo, N. Sahota, H.P. McDonald, X. Yao, Interventions for enhancing medication adherence. In Cochrane Database of Systematic Reviews 2008. 2008(2).

[3] Association, N.C.P., Take as Directed: A Prescription Not Followed. Research conducted by The Polling Company., 2006. 5(00).

[4] F.R. Ernst, A.J. Grizzle, Drug-related morbidity and mortality: updating the cost-of-illness model. In JAPA, 2001. 41(2): p. 192-199.

[5] G. Gesano, F. Heins,A. Naldini, Regional Challenges in the perspective of 2010, In Regional desparities and future challenges, 2009, ISMERI Europa.

[6] S.R. Kaufman, Construction and practice of medical responsibility: Dilemmas and narratives from geriatrics. In Culture, Medicine and Psychiatry, 1997. 21(1): p. 1-26.

[7] C. Coley et al., Preferences for home vs hospital care among low-risk patients with community-acquired pneumonia. Archives of Internal Medicine, 1996. 156(14): p. 1565.

[8] N. Mik-Meyer and K. Villadsen, Magtens former: Sociologiske blikke på statens møde med borgeren. Sociologiske perspektiver på statens møde med borgeren2007, Copenhagen: Hans Reitzels Forlag.

[9] NSI. NIS: Video about FMK. 2012; Available from: http://www.nsi.dk/sitecore/content/Nsi/Eng/English/englishFMK.asp $\mathrm{x}$ 
[10] J.R. Matsuyama, B.J. Mason, S.G. Jue, Pharmacists' interventions using an electronic medication-event monitoring device's adherence data versus pill counts. Ann Pharmacother, 1993. 27(7-8): p. 851-855

[11] M. Massimi, R.M. Baecker,M. Wu, Using participatory activities with seniors to critique, build, and evaluate mobile phones, In Proc. ACCESS 2007, ACM Press. p. 155-162.

[12] G. Dewsbury, M. Rouncefield, I. Sommerville, V. Onditi,P. Bagnall, Designing technology with older people. Universal Access in the Information Society, 2007. 6(2): p. 207-217.

[13] A. Holzinger, Finger instead of mouse: touch screens as a means of enhancing universal access, In Proc. User interfaces for all 2003, Springer-Verlag. p. 387-397.

[14] P.L.P. Rau, J.W. Hsu, Interaction devices and web design for novice older users. In Educational Gerontology, 2005. 31(1): p. 19-40.

[15] J. Häikiö, A. Wallin, M. Isomursu, H. Ailisto, T. Matinmikko,T. Huomo, Touch-based user interface for elderly users, In Proc. MobileHCI 2007, ACM Press. p. 289-296.

[16] L. Axelrod et al., The reality of homes fit for heroes: design challenges for rehabilitation technology at home. In Journal of Assistive Technologies, 2009. 3(2): p. 35-43.

[17] E. Grönvall and M. Kyng, On participatory design of home-based healthcare. Cognition, Technology \& Work, 2012: p. 1-13.

[18] R. Aarhus, S.A. Ballegaard, Negotiating boundaries: managing disease at home, In Proc. CHI 2010, ACM Press. p. 1223-1232.

[19] N. Verdezoto and J.W. Olsen, Personalized Medication Management : Towards a Design of Individualized Support for Elderly Citizens at Home, In Proc. IHI 2012, ACM Press. p. 813-817.

[20] M. Hoogendoorn, M.C.A. Klein,B. Mosch, Online application for simulating intelligent support for medicine intake, In Proc. PETRA 2008, ACM Press. p. 1-7.

[21] R.d. Oliveira, M. Cherubini, N. Oliver, MoviPill: improving medication compliance for elders using a mobile persuasive social game, In Proc. UBICOMP 2010, ACM Press. p. 251-260.

[22] T.L. Hayes, J.M. Hunt, A. Adami,J.A. Kaye, An Electronic Pillbox for Continuous Monitoring of Medication Adherence, In Proc EMBS 2006. p. 6400-6403.

[23] M.L. Lee and A.K. Dey, Reflecting on pills and phone use: supporting awareness of functional abilities for older adults, In Proc. CHI 2011, ACM Press. p. 2095-2104.

[24] E. Becker, V. Metsis, R. Arora, J. Vinjumur, Y. Xu,F. Makedon, SmartDrawer: RFID-based smart medicine drawer for assistive environments, In Proc. PETRA 2009, ACM Press. p. 1-8.

[25] Vitality - GlowCaps. 2012 [cited 20122 May]; Available from: http://www.vitality.net.

[26] PillboxApp. myCommunity Pillbox. 2011; Available from: http://pillboxapp.com/.

[27] PillBoxie. Pillboxie | The easy way to remember your meds. 2011; Available from: http://www.pillboxie.com/.

[28] ATANASSOV, N.F. MedBox. 2011; Available from: http://iphone.podnova.com/software/85035.htm.

[29] B.M. Korsch, E.K. Gozzi,V. Francis, Gaps in Doctor-Patient Communication: I. Doctor-Patient Interaction and Patient Satisfaction. In Pediatrics, 1968. 42(5): p. 855-871.
[30] L.M.L. Ong, J.C.J.M. de Haes, A.M. Hoos,F.B. Lammes, Doctorpatient communication: A review of the literature. In Social Science \& Medicine, 1995. 40(7): p. 903-918

[31] M.A. Stewart, Effective physician-patient communication and health outcomes: a review. In CMAJ, 1995. 152(9): p. 1423.

[32] D.G. Novick,K. Ward, An interaction initiative model for documentation. Proc. Documentation 2003, ACM Press. p. 80-85.

[33] T. Ni, A.K. Karlson, D. Wigdor, AnatOnMe: facilitating doctorpatient communication using a projection-based handheld device, In Proc. CHI 2011, ACM Press. p. 3333-3342.

[34] K. Kyungtae, K. Namgon, H. Sangwoo,K. JongWon. NextGeneration Collaboration Environments for Interactive Tele-medical Consultation. Proc. Biocomputation, Bioinformatics, and Biomedical Technologies, 2008.

[35] H. Le, A. van der Merwe,M. Abrahams, A Mobile Diary Application for Diabetes Management in South Africa. In Proc. SATNAC 2011.

[36] K. Siek et al., Designing a Personal Health Application for Older Adults to Manage Medications: A Comprehensive Case Study. In Journal of Medical Systems, 2011. 35(5): p. 1099-1121

[37] Y.S. Lee, J. Tullio, N. Narasimhan, P. Kaushik, J.R. Engelsma,S. Basapur. Investigating the potential of in-home devices for improving medication adherence. 2009.

[38] L. Palen,S. Aaløkke. Of pill boxes and piano benches: home-made methods for managing medication. Proc. CSCW 2006. ACM Press

[39] Bates, M., Toward An Integrated Model of Information Seeking and Searching. Graduate School of Education and Information Studies, 2003.

[40] L.G. Dalgaard, E. Grönvall, and N. Verdezoto, Accounting for Medication Particularities: Designing for Everyday Medication Management. Proc. PervasiveHealth 2013. IEEE Press.

[41] J. Greenbaum,M. Kyng, Introduction: situated design, In Design at work1992, L. Erlbaum Associates Inc. p. 1-24.

[42] N. Verdezoto,E. Grönvall, Designing a Tablet Touch-Screen Interface for Older Adults' Medication Management. Proc. workshop on How to Design Touch Interfaces for and with Older Adults, 2012: p. 4.

[43] B. Shneiderman. The eyes have it: a task by data type taxonomy for information visualizations. Proc. Visual Languages 1996.

[44] DLI. Min.medicin: Om Dansk Lægemiddel Information A/S. 2012; Available from: http://min.medicin.dk/Artikler/Artikel/58\#MASTER

[45] Sencha. Mobile JavaScript Framework. 2011; Available from: http://www.sencha.com/products/touch

[46] PhoneGap. PhoneGap. 2011; Available from: http://phonegap.com/

[47] A. Shaw. Full Calendar. 2012; Available from: http://arshaw.com/fullcalendar/.

[48] N.M. Medicine, Collaborhythm web page. 2012; Available from: http://newmed.media.mit.edu/collaborhythm

[49] D.A. Norman, Cognitive artifacts 1990: Department of Cognitive Science, University of California, San Diego

[50] C. Neustaedter, A.J.B. Brush,S. Greenberg, The calendar is crucial: Coordination and awareness through the family calendar. ACM Trans. Comput.-Hum. Interact., 2009. 16(1): p. 1-48.

[51] M. Dörk, S. Carpendale, C. Williamson, The information flaneur: a fresh look at information seeking, In Proc. CHI 2011, ACM Press. 